\title{
PROJECTIONS ALGEBRAICALLY GENERATE THE BOUNDED OPERATORS ON REAL OR QUATERNIONIC HILBERT SPACE
}

\author{
SAMUEL S. HOLLAND, JR.
}

(Communicated by Palle E. T. Jorgensen)

ABstract. We prove the theorem of the title.

Let $H$ be an infinite-dimensional, separable Hilbert space with scalars the real numbers $\mathbb{R}$ or the quaternions $\mathbb{H}$. Consider $\mathscr{B}(H)$, the *-ring of all bounded, everywhere-defined linear operators $T$ on $H$ where linear means that $T(\rho x)=\rho T(x)$ for every $x \in H$ and every $\rho \in \mathbb{R}$, resp. every $\rho \in \mathbb{H}$. In both cases $\mathscr{B}(H)$ is an algebra over $\mathbb{R}$, and our purpose here is to establish that every $T \in \mathscr{B}(H)$ equals a finite sum $\sum \rho_{i} M_{i}$ where $\rho_{i} \in \mathbb{R}$ and each $M_{i}$ is a finite product of projections. $\left(E \in \mathscr{B}(H)\right.$ is a projection when $E^{2}=E^{*}=E$.) For complex Hilbert space the theorem is known [D, Proposition 7; F-T]. Our proof actually covers all three cases.

Every nonnegative $T \in \mathscr{B}(H)$ has a unique nonnegative square root, $T^{1 / 2}$, that commutes with every $A \in \mathscr{B}(H)$ that commutes with $T$. The proof of this result presented in [R-S, $\S 104]$, ostensibly just for real and complex $H$, applies without change to the quaternionic case. Using this result, we directly deduce polar decomposition: every $T \in \mathscr{B}(H)$ can be written $T=W\left(T^{*} T\right)^{1 / 2}$ where the partial isometry $W$ satisfies $\operatorname{ker}(W)=\operatorname{ker}(T)$. In particular, applying polar decomposition to a skew operator $S=-S^{*}$, we get $S=W T$ where $T=$ $\left(-S^{2}\right)^{1 / 2} \geq 0$ and $W^{*} W=W W^{*}=E$, the projection on $\operatorname{ker}(S)^{\perp}=\operatorname{im}(S)^{\perp \perp}$. Then $U=W+(I-E)$ is unitary, $U^{*} U=U U^{*}=I$, and $S=U T$.

Our proof rests on the same matrix representation device used so efficiently by Fillmore and Topping in the complex case [F-T]; a few modifications are needed to get round the fact that $\mathbb{R}$ and $\mathbb{H}$ contain no central skew elements. $\mathscr{B}(H)$ is isomorphic as a real *-algebra to the $*$-algebra $A=M_{2}(\mathscr{B}(K))$ of all $2 \times 2$ matrices with entries from $\mathscr{B}(K)$, where $K$ is another infinite-dimensional, separable Hilbert space, correspondingly real or quaternionic. The $*$-operation in $A$ is *-transpose. So we may prove our theorem for $A$. Let $\stackrel{\circ}{A}$ stand for the real $*$-subalgebra of $A$ generated algebraically by its projections. We prove $\stackrel{\circ}{A}=A$.

Given $T \in \mathscr{B}(K), 0 \leq T \leq I$, and given unitary $U \in \mathscr{B}(K)$, one checks

Received by the editors April 8, 1994.

1991 Mathematics Subject Classification. Primary 47D25. 
easily that both

$$
P(T)=\left[\begin{array}{cc}
T & (T(I-T))^{1 / 2} \\
(T(I-T))^{1 / 2} & I-T
\end{array}\right] \text { and } Q(U)=\frac{1}{2}\left[\begin{array}{cc}
I & U \\
U^{*} & I
\end{array}\right]
$$

are projections in $A$, thus lie in $\stackrel{\circ}{A}$. Hence the matrices $P(I), 2 Q(U)-I$, and $2(P(I) Q(U)-Q(U) P(I))$ all lie in $\stackrel{\circ}{A}$. Using these matrices and noting that if $T=T^{*} \in \mathscr{B}(K)$, then $0 \leq \rho T+\sigma I \leq I$ for suitable positive real numbers $\rho$ and $\sigma$, one establishes that if $\stackrel{\circ}{A}$ contains every

$$
\left[\begin{array}{ll}
T & 0 \\
0 & 0
\end{array}\right] \text { and }\left[\begin{array}{ll}
S & 0 \\
0 & 0
\end{array}\right]
$$

where $T \in \mathscr{B}(K)$ satisfies $0 \leq T \leq I$ and $S \in \mathscr{B}(K)$ satisfies $S^{*}=-S$, then $\stackrel{\circ}{A}=A$. The first matrix is $P(I) P(T) P(I)$, so lies in $\stackrel{\circ}{A}$. For the second, write $S=U T, U U^{*}=U^{*} U=I, T \geq 0$. Then the second matrix is the product of two elements of $\stackrel{\circ}{A}$,

$$
\left[\begin{array}{ll}
S & 0 \\
0 & 0
\end{array}\right]=\left[\begin{array}{ll}
U & 0 \\
0 & 0
\end{array}\right]\left[\begin{array}{ll}
T & 0 \\
0 & 0
\end{array}\right]
$$

so also lies in $\stackrel{\circ}{A}$. Thus $\stackrel{\circ}{A}=A$.

In the complex case, Fillmore has proved the much stronger result that every $T \in \mathscr{B}(H)$ is a linear combination of 17 projections [F]. Of course this cannot be true in the real and quaternionic cases, as every real-linear combination of projections is selfadjoint.

\section{REFERENCES}

[D] J. Dixmier, Position relative de deux variétés linéaires fermées dans un espace de Hilbert, Revue. Sci. 86 (1948), 387-399.

[F] P. A. Fillmore, On sums of projections, J. Funct. Anal. 4 (1969), 146-152.

[F-T] P. A. Fillmore and D. M. Topping, Operator algebras generated by projections, Duke Math. J. 34 (1967), 333-336.

[R-S] F. Riesz and Béla Sz.-Nagy, Functional analysis, 2nd ed., Ungar, New York, 1955.

Department of Mathematics and Statistics, University of Massachusetts, Amherst, MASSACHUSETTS 01003

E-mail address: holland@math.umass .edu 